\title{
Reconstruction of Parent Austenite Grain Structure Based on Crystal Orientation Map of Bainite with and without Ausforming
}

\author{
Goro MIYAMOTO, ${ }^{1)}$ Naomichi IWATA, ${ }^{2)}$ Naoki TAKAYAMA ${ }^{2)}$ and Tadashi FURUHARA ${ }^{1)}$ \\ 1) Institute for Materials Research, Tohoku University, 2-1-1 Katahira Aoba-ku, Sendai, 980-8577 Japan. E-mail: miyamoto@ \\ imr.tohoku.ac.jp $\quad$ 2) Graduate Student, Tohoku University, 2-1-1 Katahira Aoba-ku, Sendai, 980-8577 Japan.
}

(Received on February 28, 2011; accepted on April 21, 2011)

\begin{abstract}
By using a reconstruction program recently developed by the present authors, the parent austenite grain structure is reconstructed from the crystal orientation map of bainite structure. Local austenite orientation can be reconstructed from non-ausformed bainite structure with errors of $1.7^{\circ}$ and $1.0^{\circ}$ at spatial resolutions of 5 and $20 \mu \mathrm{m}$ respectively. The angle of errors is further reduced to be less than $0.5^{\circ}$ when an analysis area is extended to a whole austenite grain. Application of the reconstruction program to the bainite structure transformed from deformed austenite has clarified that the variation in grain shape and deformation texture of parent austenite can be well reconstructed.
\end{abstract}

KEY WORDS: bainite; texture; orientation relationship; ausforming; EBSD.

\section{Introduction}

Transformation into martensite from work-hardened austenite $(\gamma)$ has been known to increase strength without significant deterioration of elongation. ${ }^{1)}$ This process has been called as ausforming. The inheritance of high density of dislocation in $\gamma$ to martensite and variations in microstructure size and transformation texture are considered to be responsible for improving mechanical properties. ${ }^{1,2)}$ The effects of this ausforming on bainite transformation have also been investigated from the view points of mechanical properties, ${ }^{3-5)}$ block or packet sizes, ${ }^{6)}$ variant selection ${ }^{7,8)}$ and transformation kinetics. ${ }^{8,9)}$

In order to understand the effects of ausforming on martensite or bainite transformation in detail, the microstructure of the parent $\gamma$, such as grain shape, crystal orientation and deformation substructure, are needed to be clarified. If the untransformed $\gamma$ is retained at room temperature, they can be analyzed directly. ${ }^{8,10}$ However, the volume fraction of retained $\gamma$ is very low in ordinary low-alloy low-carbon steels.

In order to investigate the parent grain structure, reconstruction methods have been developed by some researchers in $\mathrm{Ti}$ and $\mathrm{Zr}$ alloys ${ }^{11-15)}$ or steels. ${ }^{16-18)}$ These methods are based on the crystal orientation map, which is measured by electron back scattering diffraction (EBSD), of the product phase transformed from a non-deformed parent phase. They assume a rational orientation relationship (OR) between the parent and product phases, such as Kurdjumov-Sachs or Nishiyama-Wasserman relationships for fcc to bcc transformation, then calculate the orientation of the parent phase. In contrast, the present authors ${ }^{19)}$ have recently developed a new program to reconstruct a $\gamma$ orientation map based on an orientation map of bcc martensite, in which an OR between $\gamma$ and martensite is assumed to have non-parallel relations in close-packed planes and directions. ${ }^{20)}$ The same program was applied to ausformed martensite, and a deformed $\gamma$ structure was successfully visualized. However, the question whether the deformation in $\gamma$ that has appeared as misorientation in reconstructed $\gamma$ is due to ausforming or to plastic accommodation of transformation strain in $\gamma^{21)}$ still remains unresolved.

In this paper, effects of parameters used in the reconstruction procedure on accuracy in determining $\gamma$ orientation from non-ausformed bainite structure have been examined from the view point of orientation spread within austenite grains. Then $\gamma$ orientation maps have been reconstructed from ausformed bainite structure in order to discuss the origin of the misorientation in reconstructed $\gamma$.

\section{Experimental Procedure}

$\mathrm{Fe}-0.15 \mathrm{C}-3 \mathrm{Ni}-1.5 \mathrm{Mn}-0.5 \mathrm{Mo}$ (mass $\%$ ) alloy was used in this study. The alloys were homogenized at $1453 \mathrm{~K}$ for $259.2 \mathrm{ks}$. Cylindrical specimens, $8 \mathrm{~mm}$ in diameter and 12 $\mathrm{mm}$ in height, were cut from the homogenized specimen and heat-treated using a thermecmaster as follows. The specimens were austenitized at $1473 \mathrm{~K}$ for $0.6 \mathrm{ks}$ and subsequently compressed at $973 \mathrm{~K}$ by 10,30 or $50 \%$ at a strain rate of $1 \mathrm{~s}^{-1}$. They were then cooled by $\mathrm{He}$ gas to $673 \mathrm{~K}$, held for $3.6 \mathrm{ks}$ to isothermally transform into bainite structure and gas-quenched to room temperature. These specimens are referred to as 10, 30 and 50\%-ausformed specimens, respectively. A non-ausformed bainite specimen was also made for comparison. In order to confirm validity of reconstructed $\gamma$ orientation maps, $\gamma$-stabilized Fe-25Ni-0.5C (mass\%) alloy, which is homogenized at $1423 \mathrm{~K}$ for 345.6 ks, was also examined. The alloy was austenitized at 1323 $\mathrm{K}$ for $0.6 \mathrm{ks}$ and subsequently deformed at $973 \mathrm{~K}$ by $30 \%$ at a strain rate of $1 \mathrm{~s}^{-1}$ followed by He gas quenching. 
The ferrite $(\alpha)$ and $\gamma$ orientations were measured by EBSD at an accelerating voltage of $25 \mathrm{kV}$ and a step size of $0.5 \mu \mathrm{m}$ or $2 \mu \mathrm{m}$. Only orientation data with confidence index $(\mathrm{CI})^{22)}$ values higher than 0.1 were used for the OR determination and reconstruction of $\gamma$ orientation. The specimens for the EBSD measurements were prepared by mechanical polishing using colloidal silica, followed by $\mathrm{Ar}$ ion milling at $3 \mathrm{keV}$.

\section{Reconstruction Procedure}

In the reconstruction program developed in the previous study, ${ }^{19)}$ an $\alpha$ orientation map measured by EBSD at a certain step size is analyzed. The measured data set of $\alpha$ orientations in a small mesh, of which edge length is $L_{l}$, are here represented by the orientation matrix, $M_{i}^{B C C}$, of the ith measurement point in the mesh. In the determination of average $\gamma$ orientation in the mesh, the deviation matrix $\left(D_{i}\right)$ between the experimental and calculated $\gamma$ orientations is estimated for each $M_{i}^{B C C}$ by assuming the $\gamma$ orientation matrix $\left(M^{F C C}\right)$ and the OR matrix between bcc and fcc $\left(V^{F \rightarrow B}\right)$ as:

$$
D_{i}=V^{F \rightarrow B}\left(C_{n} M^{F C C}\right)\left(C_{m} M_{i}^{B C C}\right)^{-1}
$$

where $C_{n}$ and $C_{m}$ are the conversion operation matrices of the basic axes of cubic crystals. $C_{n}$ and $C_{m}$ are chosen for the misorientation angle of $D_{i}\left(\Delta \theta_{i}\right)$ being minimal for each i-th measurement point. The average misorientation $\left(\Delta \theta_{\text {average }}\right)$ on assuming the $\gamma$ orientation and the OR in question is given by:

$$
\Delta \theta_{\text {average }}=\sum_{N} \Delta \theta_{i} / N
$$

where $\mathrm{N}$ is the number of data in a single mesh. Finally, $M^{F C C}$ and $V^{F \rightarrow B}$ are determined by minimizing $\Delta \theta_{\text {average }}$ by numerical fitting. By changing the position of mesh centers by a given distance $\left(L_{2}\right)$ from that of a neighboring mesh and repeating the above procedure, the $\gamma$ orientation map is reconstructed.
However, the quantity of data taken from the small mesh is not sufficient to determine both the orientation of the $\gamma$ and the OR between bainite and $\gamma$. Thus, the OR is first determined by analyzing a large area of non-ausformed bainite, and then reconstruction of the $\gamma$ orientation is carried out by using the determined OR. The determined OR matrix, which is defined as the orientation matrix of a variant of bainitic ferrite in the coordinate system of parent $\gamma$, is expressed by Euler angles in Bunge's form ${ }^{22)}$ as $\left(120.72^{\circ}\right.$, $8.88^{\circ}, 195.38^{\circ}$ ). The angular deviations between $\gamma$ and bainite in close-packed planes and directions are $1.32^{\circ}$ and $2.90^{\circ}$, respectively. This OR is used throughout this study, since OR does not vary significantly by the deformation prior to the bainite transformation. ${ }^{10)}$

\section{Results and Discussions}

Since mesh size $\left(L_{l}\right)$ determines the number of $\alpha$ orientation data in each mesh, it would strongly influence the accuracy of $\gamma$ orientation determination. Therefore, first, the effects of $L_{l}$ on the accuracy were investigated by changing $L_{l}$ from 5 to $20 \mu \mathrm{m}$ with keeping a ratio of $L_{1}$ to $L_{2}$ to be two. Fig. 1(a) shows the $\alpha$ orientation map of non-ausformed bainite measured at a step size of $2 \mu \mathrm{m}$ and Figs. $1(\mathrm{~b})$ to $1(\mathrm{~d})$ are $\gamma$ orientation maps reconstructed from Fig. (a) by using different mesh size $L_{l}$. In the $\gamma$ orientation maps, $\gamma$ grain boundaries and annealing twin boundaries are represented by black and white lines, respectively. As shown in Fig. 1(b), the $\gamma$ orientation map reconstructed by using $5 \mu \mathrm{m}$ for $L_{l}$ represents $\gamma$ grains and annealing twin boundaries clearly, although it contains many black dots inside the $\gamma$ grains. These dots represent the failure in fitting, for which the value of $\Delta \theta_{\text {average }}$ obtained using Eq. (2) is larger than $5^{\circ}$. In addition, some points inside the $\gamma$ grains are misindexed as twin oriented grains to the matrix as already reported in the previous paper. ${ }^{19)}$ These errors are caused by a small number of bainite variants contained in each mesh. By increasing $L_{l}$ to be $7 \mu \mathrm{m}$ (Fig. 1(c)) and 20
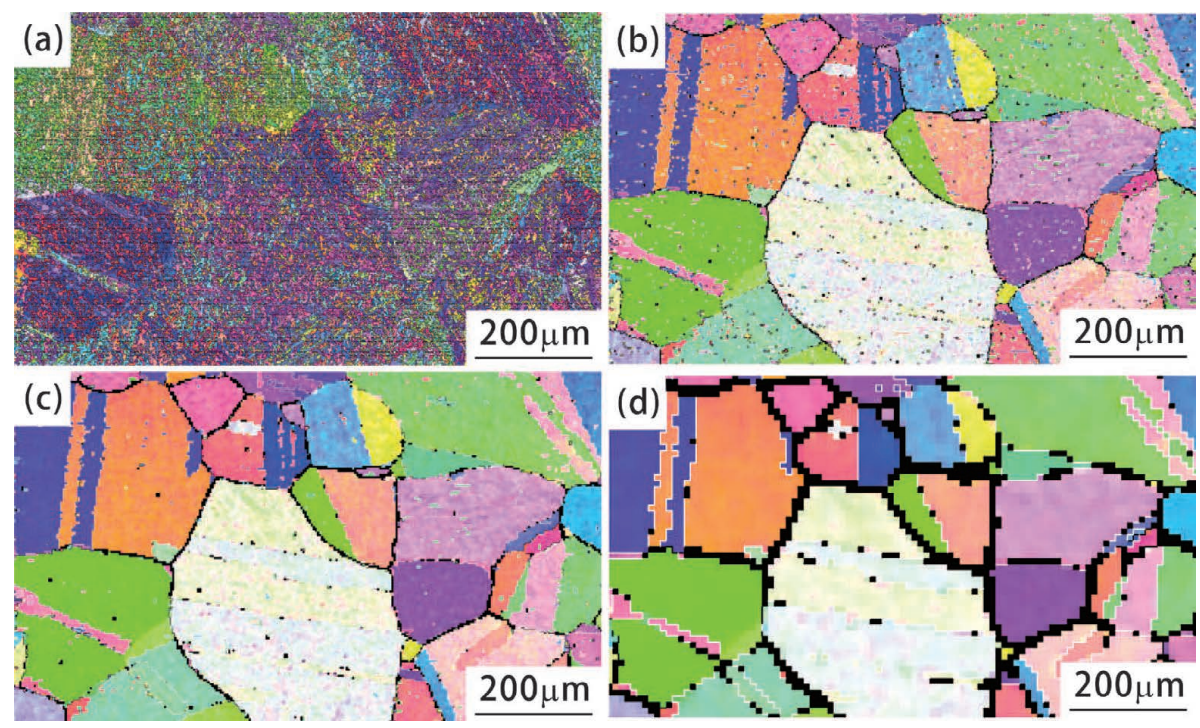

Fig. 1. (a) $\alpha$ orientation map of non-ausformed bainite measured at a step size of $2 \mu \mathrm{m}$. (b)-(d) $\gamma$ orientation maps reconstructed from (a) by using (b) $L_{1}=5 \mu \mathrm{m}$ and $L_{2}=2.5 \mu \mathrm{m}$, (c) $L_{1}=7 \mu \mathrm{m}$ and $L_{2}=3.5 \mu \mathrm{m}$, and (d) $L_{1}=20 \mu \mathrm{m}$ and $L_{2}=$ $10 \mu \mathrm{m}$. In (b), (c) and (d), black and white lines describe high angle and twin boundaries, respectively. Black dot represents failure in fitting, where the value of $\Delta \theta_{\text {average }}$ obtained using Eq. (2) is larger than $5^{\circ}$. 
$\mu \mathrm{m}$ (Fig. 1(d)), the number of failure and misindexed points decreases significantly due to increasing a number of data in each mesh, whereas spatial resolution of the orientation map becomes poorer, so that black regions corresponding to $\gamma$ grain boundaries become thicker and zigzag in alignment.

Figures 2(a) to 2(c) show $\gamma$ inverse pole figures calculated from Figs. 1(b) to 1(d) respectively. Each group of data points corresponds to orientation data from a single $\gamma$ grain. By comparing Figs. 2(a) $\left(L_{l}=5 \mu \mathrm{m}\right), 2(\mathrm{~b})\left(L_{l}=7 \mu \mathrm{m}\right)$ and 2(c) $\left(L_{l}=20 \mu \mathrm{m}\right)$, it is found that the spread of $\gamma$ orientation decreases with increasing $L_{l}$. This tendency was quantitatively analyzed by adopting the "grain orientation spread" method and is shown in Figs. 2(d) to 2(f). The grain orientation spread is obtained as follows by using $\mathrm{TSL}^{\circledR} \mathrm{OIM}$ analysis; ${ }^{23)}$ the average orientation for each $\gamma$ grain is first calculated. The spread is then the average deviation between the orientation of each point in the grain and the average orientation for the grain. As shown in Fig. 2(d), the grain spread angle is mostly distributed from $1.5^{\circ}$ to $2^{\circ}$, and its
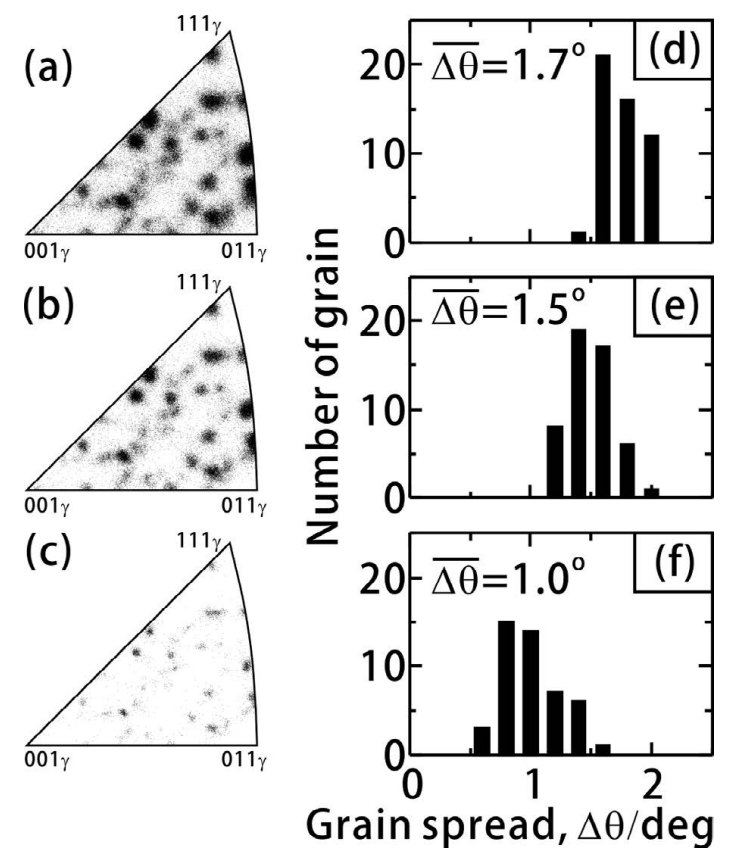

Fig. 2. Inverse pole figure of CA direction in $\gamma$ corresponding to Fig. 1, (a) $L_{l}=5 \mu \mathrm{m}$ and $L_{2}=2.5 \mu \mathrm{m}$, (b) $L_{1}=7 \mu \mathrm{m}$ and $L_{2}=$ $3.5 \mu \mathrm{m}$, and (c) $L_{1}=20 \mu \mathrm{m}$ and $L_{2}=10 \mu \mathrm{m}$. (d), (e) and (f) are distributions of grain orientation spread in $\gamma$ corresponding to (a), (b) and (c), respectively. mean value is $1.7^{\circ}$ when $5 \mu \mathrm{m}$ is used for $L_{l}$. By increasing $L_{l}$ (Figs. 2(e) and 2(f)), angles of grain spread decrease and the mean value of them reaches $1.0^{\circ}$ at $L_{l}$ of $20 \mu \mathrm{m}$ (Fig. 2(f)).

In order to achieve better accuracy for the determination of parent $\gamma$ orientation, the quantity of orientation data for the analysis was increased by decreasing the step size in EBSD measurement and increasing the area for the analysis. Figure 3(a) shows the $\alpha$ orientation map of a non-ausformed bainite specimen measured at the step size of 0.5 $\mu \mathrm{m}$. A single $\gamma$ region without containing annealing twins was selected manually from this map and divided into two regions as indicated by the regions A and B. Figures 3(b) and 3(c) show $\{001\}_{\alpha}$ pole figures measured for the regions A and B in Fig. 3(a), on which the estimated $<001\rangle_{\alpha}$ of bainite by fitting for each region are superimposed. The number of orientation data to determine one $\gamma$ orientation increases from about one hundred (Fig. 1(d)) to tens of thousands. The $<001>_{\alpha}$ directions for the regions A and B shown in Figs. 3(b) and 3(c) appear quite similar, thus the $<001>_{\gamma}$ directions obtained by the fitting are also nearly the same in the two regions. The misorientation angle of the parent $\gamma$ calculated between the regions A and B is $0.74^{\circ}$. Such analyses were carried out for more than ten different $\gamma$ regions and the mean misorientation angle between two regions divided is obtained to be $0.65^{\circ}$. Since the misorientation between two regions includes errors arising in determination of orientations for both regions, it is concluded that the orientation of each region can be determined with an average error of $0.33^{\circ}$. Such accurate determination of the parent $\gamma$ orientation has been recently applied to analyze OR between reversely formed $\gamma$ and pearlitic $\alpha$ in high carbon steel ${ }^{24)}$ or between $\gamma$ and grain boundary $\alpha$ in medium carbon steels. ${ }^{25)}$

Figures 4(a), 4(b) and 4(c) show $\gamma$ orientation maps reconstructed from $\alpha$ orientation maps of ausformed bainite, of which deformation strains applied to $\gamma$ are $10 \%, 30 \%$ and $50 \%$ respectively. After trial and error, $7 \mu \mathrm{m}$ and $3.5 \mu \mathrm{m}$ were chosen for $L_{1}$ and $L_{2}$, respectively, in reconstruction of $\gamma$ from ausformed bainite because deformation structures in $\gamma$ reconstructed by using these values were clearer than other conditions investigated. The compression axis (CA) was taken as the reference axis for the color orientation maps according to the stereographic triangle shown in Fig. 4(a). As shown in Fig. 4(a), the deformation by $10 \%$ hardly changes $\gamma$ grain shape although a gradual lattice rotation of $\gamma$ is partly observed as indicated by black arrows. By

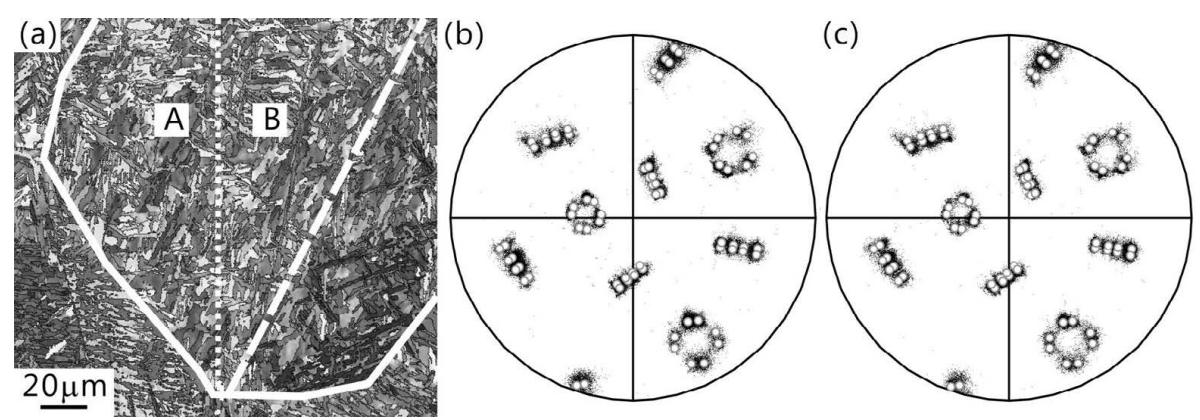

Fig. 3. (a) $\alpha$ orientation map of non-ausformed bainite structure measured at a step size of $0.5 \mu \mathrm{m}$ and $\{001\} \alpha$ pole figures taken from the regions (b) A and (c) B, on which $<001>_{\alpha}$ directions obtained by fitting are superimposed. Solid and broken lines in (a) indicate prior $\gamma$ grain boundaries and annealing twin boundaries, respectively. 

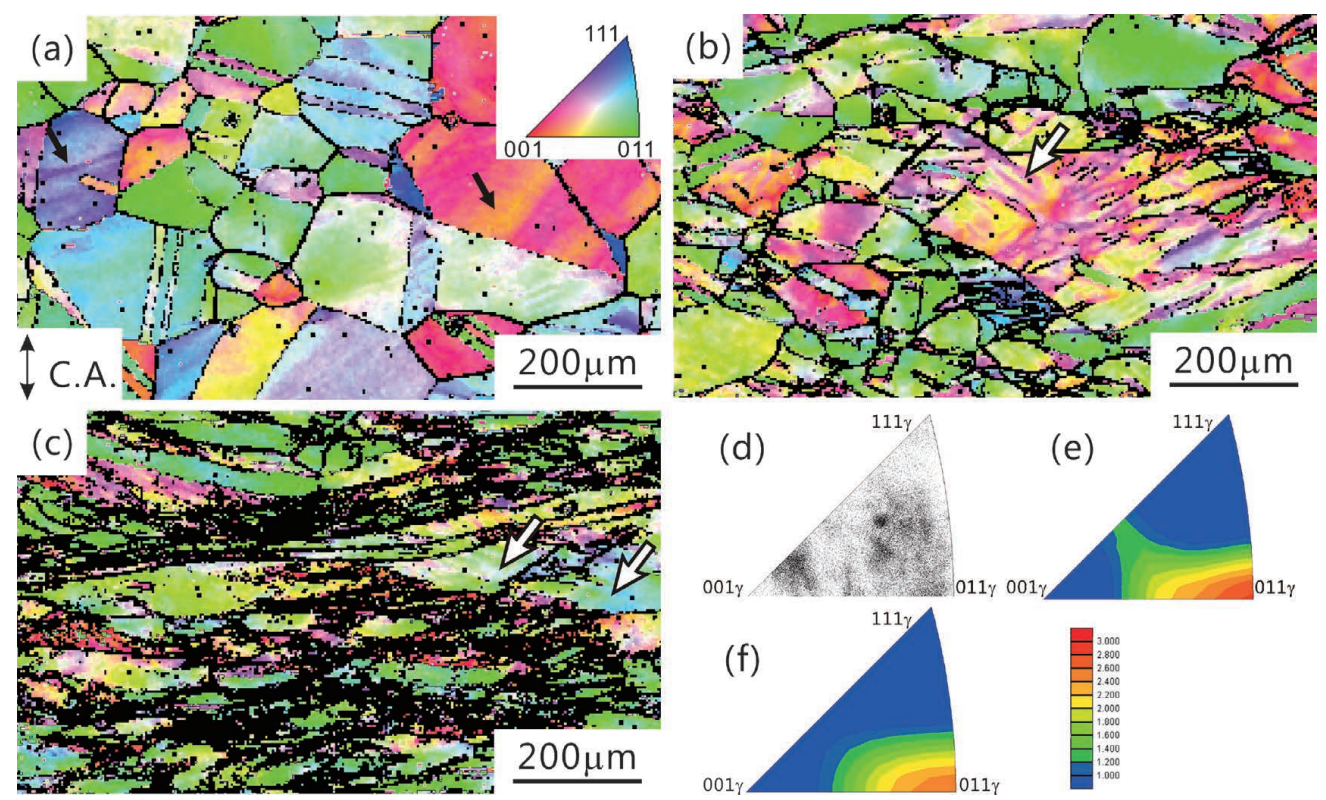

Fig. 4. Orientation maps of $\gamma$ reconstructed from (a) 10\%-, (b) 30\%-, (c) 50\%-ausformed bainite specimens, and (d), (e), (f) inverse pole figures of CA direction in $\gamma$ corresponding to (a), (b), (c), respectively. $L_{1}$ and $L_{2}$ used are $7 \mu \mathrm{m}$ and $3.5 \mu \mathrm{m}$, respectively. Details of $\gamma$ orientation map are described in the caption of Fig. 1.
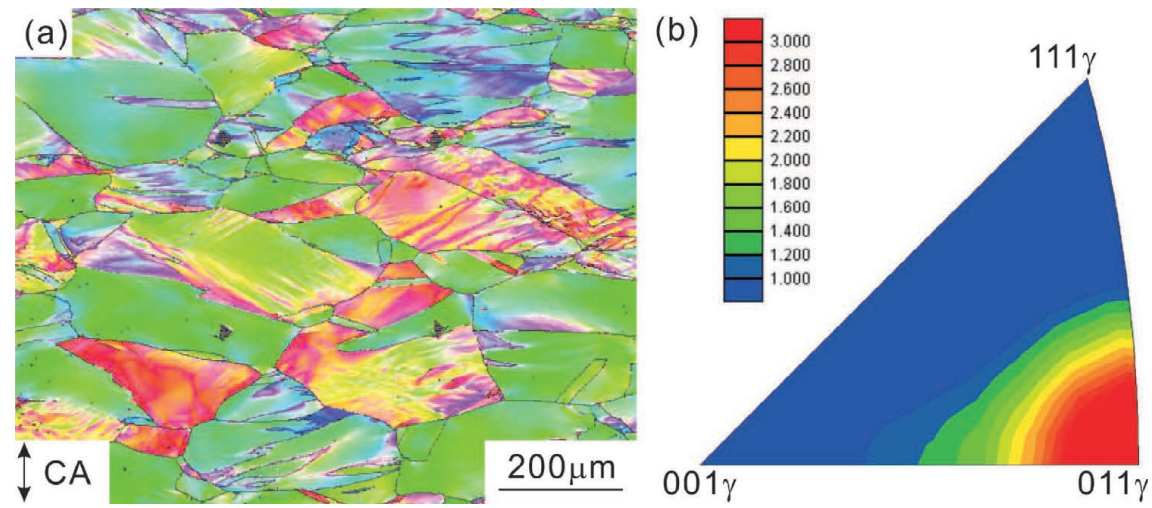

Fig. 5. (a) Measured orientation map of $\gamma$ in the $\mathrm{Fe}-25 \mathrm{Ni}-0.5 \mathrm{C}$ alloy deformed at $973 \mathrm{~K}$ by $30 \%$ ( step size $; 2 \mu \mathrm{m}$ ) and (b) inverse pole figure of CA direction in $\gamma$. CA was taken as the reference axis for the color orientation maps according to a stereo triangle shown in Fig. 4(a). Details of $\gamma$ orientation map are described in the caption of Fig. 1.

increasing the deformation strain to $30 \%$ (Fig. 4(b)) and $50 \%$ (Fig. 4(c)), $\gamma$ grains become elongated perpendicularly to $\mathrm{CA}$, and orientation change inside the $\gamma$ grains becomes substantial. As indicated by open arrows, banded structures inclined by about $45^{\circ}$ to the CA direction are also seen. The frequency of failure in reconstruction represented by black points increases with strain because a large and local misorientation in $\gamma$ introduced by the deformation results in increase in $\Delta \theta_{\text {average }}$ in Eq. (2). Figure 4(d) represents the inverse pole figure for the CA direction in $\gamma$ reconstructed for the $10 \%$ ausformed bainite specimen, which corresponds to Fig. 4(a). By comparing it with Fig. 2(b), it is clearly seen that the orientation spread of each $\gamma$ grain becomes much larger by the deformation of $10 \%$. The crystal lattice of $\gamma$ tends to rotate into a specific direction by further deformation, causing a development of deformation texture. The $\gamma$ inverse pole figures calculated from 30\%- (Fig. 4(e)) and 50\%- (Fig. 4(f)) ausformed specimens clearly indicate that the $\left\langle 011>_{\gamma}\right.$ component becomes much stronger than the $<111>_{\gamma}$ and $<001>_{\gamma}$ components.
In order to confirm the validity of $\gamma$ orientation map reconstruction, deformation structure in the $\gamma$-stabilized alloy was examined. Figures 5(a) and 5(b) show the $\gamma$ orientation map of the $\mathrm{Fe}-25 \mathrm{Ni}-0.5 \mathrm{C}$ alloy deformed at $973 \mathrm{~K}$ by $30 \%$ and the inverse pole figure of CA direction in $\gamma$, respectively. Elongated $\gamma$ grains and banded structures inclined by about $45^{\circ}$ to CA are observed, which is similar to those in the reconstructed $\gamma$ orientation map in Fig. 4(b). It was found by trace analyses that these bands tended to be introduced along $\{111\}_{\gamma}$ planes. Such deformation band structures were similarly observed in a hot-rolled austenitic stainless steel. ${ }^{26)}$ Furthermore, the deformation texture of $\gamma$ shown in Fig. 5(b) agrees quite well with that reconstructed (Fig. 4(e)), although the intensity of $\langle 011\rangle_{\gamma}$ peak in Fig. 5(b) is higher than that in Fig. 4(e). Such deformation texture of $\gamma$ coincides also well with the deformation texture reported in fcc metal after compression rolling ${ }^{27)}$ and in $\gamma$ of the $\mathrm{Fe}-30 \% \mathrm{Ni}$ specimen compressed at $573 \mathrm{~K}^{28)}$

Displacive transformation such as martensite and bainite transformations causes a lattice rotation in surrounding $\gamma$ 
due to accommodation of transformation strain. ${ }^{21)}$ However, the present reconstruction method reproduces the spreading of $\gamma$ orientation in each $\gamma$ grain and the development of deformation texture by ausforming successfully. This fact clearly supports that the lattice rotation in $\gamma$ due to the ausforming can be reconstructed by the present method. Therefore, whether the misorientation in $\gamma$ reconstructed from ausformed specimens is due to ausforming or to accommodation of transformation strain should be the subject under discussion only at a small applied strain less than $10 \%$. By comparing the orientation spread in $\gamma$ reconstructed from the $10 \%$-ausformed specimen (Fig. 4(d)) with the orientation spread in the non-ausformed specimen (Fig. 2(b)), it is further concluded that the contribution of plastic accommodation of transformation strain to the misorientation in reconstructed $\gamma$ is smaller than that by ausforming even at the strain of $10 \%$.

\section{Summary}

In the present study, the reconstruction method of parent $\gamma$ orientation, which has been developed by the present authors, ${ }^{19)}$ was applied to analyze bainite structure with and without ausforming. In application to the non-ausformed specimen, it was found that the local orientation of $\gamma$ could be obtained with errors $1^{\circ}$ by using a large square analysis area of $20 \mu \mathrm{m}$ in side length. When the analysis area is extended to a whole $\gamma$ grain, such angle of errors decreases less than $0.5^{\circ}$. In the application to the ausformed bainite structure, $\gamma$ grains elongated perpendicularly to the compression axis and deformation texture in $\gamma$ were reconstructed. These features in reconstructed $\gamma$ agree well with those observed in a $\gamma$-stabilized alloy deformed at the same condition.

\section{Acknowledgement}

The authors gratefully acknowledge financial support from the Research Promotion Fund of the Iron and Steel Institute of Japan and the Ministry of Education, Culture, Sports, Science and Technology through a Grant in Aid for Young Scientists (B) No. 21760526 (2009-2010).

\section{REFERENCES}

1) I. Tamura: Trans. ISIJ., 27 (1987), 763.

2) I. Tamura: Trans ISIJ., 6 (1966), 249.

3) D. Kalish , S. A. Kulin and M. Cohen: J. Metals., 17 (1965), 157.

4) R. H. Edwards and N. F. Kennon: Metall. Trans. A, 9A (1978), 1801.

5) K. Fujiwara and S. Okaguchi: Tetsu-to-Hagané, 80 (1994), 771.

6) H. Kawata, K. Sakamoto, T. Moritani, S. Morito, T. Furuhara and T. Maki: Mater. Sci. Eng. A., 438-440 (2006), 140.

7) A. A. Shirzadi, H. Abreu, L. Pocock, D. Klobcar, P. J. Withers and H. K. D. H. Bhadeshia: Int. J. Mater. Res., 100 (2009), 40.

8) W. Gong, Y. Tomota, M. S. Koo and Y. Adachi: Scripta Mater., 63 (2010), 819.

9) R. Freiwillig, J. Kudrman and P. Chraska: Metall. Mater. A, 7A (1976), 1091.

10) H. Regle, N. Maruyama and N. Yoshinaga: Proc. of Int. Conf. on Advanced High Strength Sheet Steels for Automotive Applications, ed. by M. A. Baker, AIST, Warrendale, PA, (2004), 239.

11) M. Humbert, F. Wagner, H. Moustahfid and C. Esling: J. Appl. Crystallogr., 28 (1995), 571.

12) M. Humbert and N. Gey: J. Appl. Crystallogr., 35 (2002), 401.

13) N. Gey and M. Humbert: J. Mater. Sci., 38 (2003), 1289.

14) M. G. Glavicic, P. A. Kobryn, T. R. Bieler and S. L. Semiatin: Mater. Sci. Eng. A, 346A (2003), 50.

15) M. G. Glavicic, P. A. Kobryn, T. R. Bieler and S. L. Semiatin: Mater. Sci. Eng. A, 351A (2003), 258.

16) C. Cayron, B. Artaud and L. Briottet: Mater. Charact., 57 (2006), 386.

17) T. Morimoto, F. Yoshida, I. Chikushi, H. Kitahara and N. Tsuji: Tetsu-to-Hagané, 93 (2007), 591.

18) P. Blaineau, L. Germain, M. Humbert and N. Gey: Solid State Phen., 160 (2010), 203.

19) G. Miyamoto, N. Iwata, N. Takayama and T. Furuhara: Acta Mater., 58 (2010), 6393.

20) G. Miyamoto, N. Takayama and T. Furuhara: Scripta Mater., 60 (2009), 1113.

21) G. Miyamoto, A. Shibata, T. Furuhara and T. Maki: Acta Mater., 57 (2009), 1120.

22) A. J. Schwartz, M. Kumar, B. L. Adams and D. P. Field: Electron Backscattered Diffraction in Materials Science 2nd ed., Springer, New York, (2009), 45.

23) Orientation Imaging Microscopy software version V5.2, user manual, TSL, Draper, UT.

24) Z.-D. Li, G. Miyamoto, Z.-G. Yang and T. Furuhara: Scripta Mater., 60 (2009), 485.

25) G. Miyamoto, B. Poorganji and T. Furuhara: Solid State Phen., in press.

26) P. Cizek, J. A. Whiteman, W. M. Rainforth and J. H. Beyon: J. Microscopy, 213 (2004), 285.

27) C. S. Barrett and L. H. Levenson: Trans. AIME, 137 (1940), 112.

$28)$ N. J. Wittridge, J. J. Jonas and J. H. Root: Metall. Mater. Trans. A, 32A (2001), 889. 